\title{
Sexual dysfunction among women with systemic lupus erythematosus: might it reflect cardiovascular disease burden?
}

\author{
Dimitrios Ioannis Patoulias \\ $1^{\text {st }}$ Department of Internal Medicine, Hippokration General Hospital, Thessaloniki, Greece
}

\section{Dear Editor,}

In issue no. 1/2019 of the journal Reumatologia, Moghadam ZB et al. in the article "Prevalence of sexual dysfunction in women with systemic lupus erythematosus and its related factors" demonstrated that even young women with systemic lupus erythematosus (SLE), with a median disease duration of less than 10 years and inactive or moderately active disease, are more prone to sexual dysfunction compared to healthy controls (85.9\% vs. $26.5 \%$ ) [1]. Age and disease activity arise as major determinants of sexual dysfunction, whereas this aspect is usually underrated by healthcare physicians.

Besides its psychosocial importance, sexual function has been shown to be closely related to vascular disease in patients with SLE, and vice versa; in their cross-sectional analysis, Tseng et al. showed that vascular disease increased the odds for impaired sexual function by 5.7 times among sexually active female patients with SLE (95\% confidence interval [CI] 1.6-20.1), representing the only significant determinant of sexual dysfunction [2].

It is well established that cardiovascular disease (CVD) is associated with sexual dysfunction, both in male and female patients [3]. Endothelial dysfunction might play a crucial role in the development of so-called "vasculogenic" female sexual dysfunction [4]. Patients with SLE, even those who are free of CVD, have impaired endothelial function, with diabetes mellitus and renal disease constituting major contributors [5].

It may therefore be deduced that sexual function represents another clinical marker of subclinical vascular disease among patients with SLE. Based on the fact that patients with SLE feature an increased cardiovascular burden, both in terms of subclinical atherosclerosis [6] and major adverse cardiovascular events (with age and disease activity showing only a slight correlation) [7], it may be concluded that meticulous assessment of female sexual function in SLE patients can offer significant prognostic value as far as primary prevention is concerned, further directing diagnostic and therapeutic interventions.

The author declares no conflict of interest.

\section{References}

1. Moghadam ZB, Rezaei E, Faezi ST, et al. Prevalence of sexual dysfunction in women with systemic lupus erythematosus and its related factors. Reumatologia 2019; 57: 19-26.

2. Tseng JC, Lu LY, Hu JC, et al. The impact of systemic lupus erythematosus on women's sexual functioning. I Sex Med 2011; 8: 3389-3397.

3. McCabe MP, Sharlip ID, Lewis R, et al. Risk Factor for Sexual Dysfunction Among Women and Men: A Consensus Statement From the Fourth International Consultation on Sexual Medicine 2015. J Sex Med 2016; 13: 153-167.

4. Steinke EE. Sexual Dysfunction in Women With Cardiovascular Disease. J Cardiovasc Nurs 2010; 25: 151-158.

5. Mak A, Kow NY, Schwarz H, et al. Endothelial dysfunction in systemic lupus erythematosus - a case-control study and an updated meta-analysis and meta-regression. Sci Rep 2017; 7: 7320.

6. Wu GC, Liu HR, Leng RX, et al. Subclinical atherosclerosis in patients with systemic lupus erythematosus: A systemic review and meta-analysis. Autoimmun Rev 2016; 15: 22-37.

7. Ballocca F, D'Ascenzo F, Moretti C, et al. Predictors of cardiovascular events in patients with systemic lupus erythematosus (SLE): a systematic review and meta-analysis. Eur J Prev Cardiol 2015; 22: 1435-1441. 